\title{
Características de comunidades de pratica existentes no Serviço de Informação em Arte: o caso da REDARTE/RJ
}

http://dx.doi.org/10.1590/1981-5344/2696

O presente artigo é relato de pesquisa que busca identificar e analisar a existência das principais características de comunidades de prática na Rede de Bibliotecas e Centros de Informação em Arte no Estado do Rio de Janeiro - REDARTE/RJ. Utilizam-se como critério as principais características de comunidades de prática: o domínio, a comunidade e a prática, bem como os indicadores de comunidade para analisar $o$ funcionamento, as interações, o processo de troca e compartilhamento de experiências e conhecimento entre os profissionais de informação em Arte integrantes da REDARTE/RJ. A abordagem adotada foi o estudo de caso por meio de pesquisa qualitativa. Comprova-se que a REDARTE/RJ apresenta as características de comunidade de prática com os benefícios em se tratando de cooperação e interação para gestão do conhecimento. 
Palavras-chave: Comunidades de prática. Gestão do conhecimento. Redes de bibliotecas. Rede de Bibliotecas e Centros de Informação em Arte no Estado do Rio de Janeiro. REDARTE/RJ.

\section{Characteristics of existing communities of practice in the Information in Art Service: the case of REDARTE/RJ}

This article reports research that seeks to identify and analyze the existence of the main characteristics of communities of practice in the Rede de Bibliotecas e Centros de Informação em Arte no Estado do Rio de Janeiro - REDARTE/RJ. It applied the parameters: the domain, the community and the practice and indicators to analyze the operation, the interactions, the process of exchange and sharing of experiences and knowledge among members of REDARTE/RJ. The approach adopted was the case study through qualitative research. It proves that the REDARTE / RJ presents the characteristics community of practice with the benefits when it comes to cooperation and interaction for knowledge management.

Keywords: Communities of practice. Knowledge management. Library network. Rede de Bibliotecas e Centros de Informação em Arte no Estado do Rio de Janeiro. REDARTE/RJ

Recebido em 01.02.2016 Aceito em 06.05.2019

\section{Introdução}

Entende-se que o processo de Gestão do Conhecimento visa identificar, gerar, captar, registrar, disseminar, compartilhar o conhecimento tácito das empresas/organizações/instituições e colaborar com a cultura/memória organizacional. (IFLA. Knowledge Management Section).

Em pesquisa sobre Gestão do Conhecimento no site de Terra (2007, p. 57) verifica-se que para este autor a Gestão do Conhecimento utiliza ferramentas/instrumentos e práticas gerenciais, de forma a incentivar o compartilhamento de conhecimentos e a troca de experiência entre as pessoas de uma organização. $E$, que nas comunidades de prática, a ênfase está no compartilhamento de conhecimento, informações e experiências. 
Assim, comunidades de prática seriam descritas como uma das práticas de Gestão do Conhecimento onde um grupo de pessoas de uma mesma área de conhecimento compartilha uma preocupação, uma ideia, as melhores práticas e que seus membros interagem regularmente para aprender a desempenhar melhor sua função (WENGER, 2000).

O estudo contextualiza comunidade de prática como uma categoria de análise e ferramenta de Gestão do Conhecimento que possibilita organizar, intensificar a transferência de conhecimento organizacional, cooperar e a interação entre profissionais de determinada área de conhecimento. Busca identificar e analisar a existência das principais características de comunidades de prática: o domínio, a comunidade e a prática na-Rede de Bibliotecas e Centros de Informação em Arte no Estado do Rio de Janeiro - REDARTE/RJ. Indica atividades de comunidades de práticas que podem ser adotadas pela rede para dinamizar suas ações.

Os profissionais da informação que trabalham em instituições de Arte procuram atender às necessidades informacionais de seus usuários e buscam realizar seu trabalho da melhor forma possível. A carência informacional das bibliotecas e centros de informação das instituições de Arte é uma constante na realidade brasileira dos profissionais de informação. Esta deficiência estimulou a criação da REDARTE/RJ.

A rede foi idealizada por Solange Zuñiga, em 1995, então Diretora do Departamento de Pesquisa e Documentação da Fundação Nacional de Artes (FUNARTE), e que convida Helena Dodd Ferrez, Coordenadora do mesmo departamento para assumir a coordenação do grupo em formação. Ambas tinham uma preocupação - o estado de carência de documentação informacional com as quais os bibliotecários e profissionais de informação em Arte e Cultura conviviam em suas instituições, frente à necessidade de atender os seus usuários (pesquisadores, estudantes e interessados na área). Onze instituições enviaram seus representantes para a primeira reunião em dezembro daquele ano. Os anos seguintes são de muito trabalho e a rede informal conquista força e reconhecimento entre os profissionais da informação e suas organizações. Em 2006 a rede é oficialmente caracterizada como uma associação civil de natureza cultural, sem fins lucrativos, sendo uma de suas finalidades promover o acesso do público interessado em Arte aos itens informacionais, em qualquer suporte ou meio eletrônico, existentes nas Unidades Integrantes da REDARTE/RJ, respeitando a disponibilidade de cada uma. As bibliotecas e centros de informação contemplam as áreas temáticas Arquitetura e Urbanismo, Artes Decorativas, Artes Gráficas, Artes Plásticas, Cinema, Circo, Dança, Desenho Industrial, Folclore \& Cultura Popular, Fotografia (arte, técnica e história), Moda e Indumentária, Música, Numismática, Ópera, Rádio, Teatro, TV, Vídeo.

Constata-se que a Rede pode ser considerada uma comunidade de prática e pode se apropriar de determinadas técnicas de comunidades de prática com a finalidade de potencializar suas atividades.

A estratégia metodológica utilizada neste estudo caracteriza-se como uma pesquisa de natureza exploratória e descritiva com abordagem qualitativa. Para analisar a evolução da REDARTE/RJ, seus produtos e 
serviços, recorre-se ao método qualitativo com aplicação de questionário e entrevistas. A adoção por estudo de caso como método de pesquisa ocorre por ter como hipótese de trabalho, que a REDARTE/RJ se encaixa na categoria de Comunidade de Prática por ser um grupo específico, daí a busca por identificar e analisar as principais características de comunidades de prática.

Este artigo possui a seguinte estrutura, além da presente introdução. Na seção 2 é apresentado o referencial teórico acerca de comunidades de prática utilizado na análise. Na seção 3 é explicitada à metodologia utilizada para a pesquisa. A seção 4 é formada pela análise do estudo de caso, seguida pelas considerações finais.

\section{Comunidades de prática: características e indicadores}

De acordo com Melo (2015) os conceitos fundamentais relativos às comunidades de prática foram encontrados em diferentes trabalhos de autores como Etienne Wenger (1998, 2000, 2001, 2006, 2010), Richard McDermott (2001) e Etienne Wenger, Richard McDermott e William Snyder (2002).

O conceito de comunidade de prática tem sua origem na tentativa de explicar a natureza social da aprendizagem humana inspirada pela antropologia e teoria social, segundo estudos realizados por Wenger e Lave no final dos anos 80, conforme relato de Wenger (2010, p. 1).

Segundo Wenger (2010, p. 1) o conceito de comunidade de prática não existe por si só. A concepção de CoP precisa ser contextualizada, isto é,

faz parte de um conceito mais amplo, trata-se de estrutura para pensar sobre a aprendizagem em suas dimensões sociais. É uma perspectiva que localiza aprendizagem, não na cabeça ou no exterior, mas na relação entre a pessoa e o mundo, que para os seres humanos é um ser social em um mundo social. (WENGER, 2010, p. 1). práticas

Para Wenger, McDermott e Snyder (2002, p. 4) comunidades de

são grupos de pessoas que compartilham um interesse ou "paixão" por determinado assunto que pretende desenvolver competências de seus participantes de forma a fazer e aprender fazê-lo de um modo melhor".(WENGER; MCDERMOTT; SNYDER, 2002, p. 4).

Dessa forma, entende-se que as CoPs geram e trocam conhecimento.

Quais são os atributos pilares de comunidades de prática? 0 domínio, a comunidade e a prática é a resposta, mas Wenger, McDermott e Snyder (2002, p. 27) mencionam que somente esses três elementos não definem a participação de uma pessoa em uma comunidade. Alguns indivíduos tomam parte por quererem cuidar do domínio e por desejarem ver o seu desenvolvimento; outros, por considerarem o valor da participação e pela maior interação com os seus pares. 
De acordo com os autores, O domínio - o embasamento é comum a todos. Com o passar do tempo é o que institui uma identidade para o grupo e define o foco da discussão na comunidade. Legitima a comunidade por afirmar seus propósito e valor para os membros e as partes interessadas. Existe uma competência compartilhada que diferencia seus membros de outros. É algo dinâmico que se atualiza com o mundo social e a própria comunidade.

A comunidade - é constituída pelo grupo de pessoas que interagem, colaboram, compartilham informações e conhecimento. Estabelecem relações de forma a aprenderem um com os outros. Desenvolvem um sentimento de pertencer a algo, de estar engajadas em algo que contribua para melhoria de suas atividades. O grupo apreende junto e constrói em conjunto, o que pode resultar em produtos e serviços. A interação cria uma "common history and communal identity", mas isto não quer dizer que sempre exista harmonia. Daí a importância do líder de uma comunidade estar preparado para acompanhar a evolução da mesma.

A prática - os membros de uma comunidade de prática são os praticantes. Desenvolvem um repertório compartilhado de recursos: experiências, ideias, histórias, ferramentas, estilo e formas de lidar com problemas recorrentes, documentos compartilhados pelos membros. Isto leva tempo e interação sustentada. Os participantes estabelecem rotinas para organização do conhecimento de forma que possa ser útil para cada um em suas unidades. E o resultado é um produto da comunidade e não do indivíduo.

Observa-se que 0 ambiente de comunidade de prática pode aprimorar o conhecimento de uma coletividade, isto é, trata-se de propiciar ao aprendizado e a geração de novos conhecimentos.

Nonaka e Takeuchi (1997, p. 65) classificam em dois segmentos a criação de conhecimento. No primeiro segmento "o conhecimento é um processo criado por pessoas". Os autores afiançam que

Uma organização não pode criar conhecimento sem indivíduos. A organização apoia os indivíduos criativos ou lhes proporciona contexto para a criação de conhecimento. A criação de conhecimento organizacional, pois, deve ser entendida como um processo que amplia "organizacionalmente" o conhecimento criado pelos indivíduos, cristalizando-o como parte da rede de conhecimento da organização. Esse processo ocorre dentro de uma 'comunidade de interação' em expansão, que atravessa níveis e fronteiras interorganizacionais. (NONAKA; TAKEUCHI, 1997, p. 65).

O segundo segmento fundamenta-se na "distinção estabelecida entre conhecimento tácito e conhecimento explícito". Onde o conhecimento tácito é aquele que é pessoal, intrínseco às experiências de vida de cada indivíduo e, assim, difícil de ser formulado e comunicado Polanyi (1966) apud Nonaka e Takeuchi (1997, p. 65).

Enquanto que o conhecimento explícito ou "codificado", pode ser encontrado num ambiente estruturado, refere-se ao conhecimento transferível em linguagem formal e sistemática. (NONAKA; TAKEUCHI, 1997, p. 65). 
Os autores ressaltam a existência de distinção entre conhecimento tácito e explicito, mas alertam que os mesmos têm conexões. Segundo eles, "[...] o conhecimento tácito e o conhecimento explícito não são entidades totalmente separadas e, sim mutuamente complementares. Interage um com o outro e realizam trocas nas atividades criativas dos seres humanos". (NONAKA; TAKEUCHI, 1997, p. 67).

A descrição de comunidades de prática ressalta que se constituem em um grupo no qual os participantes não necessariamente trabalham juntos, estão dispersos em áreas geograficamente distintas, mas se encontram, porque percebem o valor dessas interações. Com isso, aumenta a satisfação em estar e conhecer colegas que compartilham perspectivas e problemas semelhantes. Com o tempo os participantes criam uma identidade própria, desenvolvem relações de caráter mais pessoal (confiança) e estabelecem mecanismos de interação. (WENGER; MCDERMOTT; SNYDER, 2002).

É importante salientar que o gestor de uma área/departamento/empresa quando inicia o planejamento para adotar uma comunidade de prática como instrumento de organização do conhecimento organizacional deve atentar para determinados indicadores. Wenger (1998, p.125-126) elenca 14 elementos determinantes para estruturar uma CoP. Enquanto Pinho (2011, p. 65) com base em Wenger estabelece em sua pesquisa 19 indicadores que configuram uma comunidade de prática.

Seguem os indicadores, segundo Wenger (1998):

1. As relações mútuas sustentáveis - harmoniosas ou conflituosas;

2. Formas compartilhadas de engajar-se em fazer as coisas juntos;

3. O rápido fluxo de informações e propagação da inovação;

4. Ausência de preâmbulos introdutórios, como se conversas e interações foram apenas a continuação de um processo contínuo;

5. Configuração muito rápida de um problema a ser discutido;

6. Sobreposição substancial entre as descrições de quem pertence dos participantes;

7. Conhecer o que os outros sabem o que eles podem fazer, e como eles podem contribuir para uma empresa;

8. Identidades mutuamente definidoras;

9. A capacidade de avaliar a adequação das ações e produtos;

10. Ferramentas específicas, representações e outros artefatos;

11. A sabedoria local, histórias compartilhadas, piadas, sabendo riso; 
12. Jargão e atalhos para as comunicações, bem como a facilidade de produzir novos;

13. Certos estilos reconhecidos como a indicação de membros;

14. Um discurso compartilhado refletindo certa perspectiva sobre o mundo.

Nota-se que os indicadores enumerados por Wenger (1998, p. 125126) e as três características de comunidades de práticas definidas Wenger, McDermott e Snyder (2002, p. 27) são complementares. Neste sentido, buscou-se identificar e analisar estas características nas atividades, produtos e serviços da REDARTE/RJ.

\section{Metodologia}

O presente artigo está referenciado em pesquisa realizada no âmbito do Programa de Pós-graduação em Biblioteconomia da UNIRIO, que resultou na dissertação de mestrado de uma das autoras com o objetivo de identificar e analisar a existência das principais características de comunidades de prática nas atividades da REDARTE/RJ.

Trata-se de um estudo de caso onde foram selecionados aspectos que representam características de comunidades de práticas no resultado da análise dos dados coletados no decorrer da pesquisa mencionada acima.

Devido ao volume do material coletado, optou-se por construir um quadro com as características e indicadores de comunidades de prática comparados aos resultados das assertivas respondidas pelos membros da rede.

Apresenta-se o Gráfico1 com uma síntese das características de comunidade de prática (o Domínio, a Comunidade e a Prática) existentes nas atividades, produtos e serviços da REDARTE/RJ.

\section{Identificação e análise das características de comunidades de prática na REDARTE/RJ}

O objetivo principal do presente estudo é identificar e analisar a existência das principais características de comunidades de prática nas atividades da REDARTE/RJ e indicar atividades de comunidades de práticas que podem ser adotadas pela rede para dinamizar suas ações. No caso do presente trabalho, procurou-se operacionalizar a dimensão social do conceito de acordo com Wenger (2010) por meio da análise desta rede e dos profissionais da informação que representam suas instituições.

Esta análise baseia-se nas informações coletadas por meio de entrevistas, questionários e documentos do material da dissertação de uma das autoras. A seguir serão descritos parte dos resultados alcançados por meio de questionários para os membros, avaliação de atas de 
reuniões, entrevistas com a gestora (2012-2014) e a coordenadorafundadora da rede, conforme pesquisa realizada por Melo (2015).

Os dados coletados tanto com a aplicação do questionário, quanto às entrevistas e análise documental foram interpretados utilizando a perspectiva teórica de Wenger, McDermott e Snyder (2002) para três características (o domínio, a comunidade e a prática); Wenger (1998) para os indicadores de CoP e McDermott (2001) para os fatores críticos de sucesso de comunidades de prática.

Para cada assertiva incluída no questionário foi dada ao respondente cinco opções de escolha - concordo totalmente, concordo, desconheço, discordo e discordo totalmente.

Verificou-se que 0 perfil dos respondentes que atenderam a pesquisa foi de bibliotecários (22) e que os profissionais estão em sua maioria próxima à aposentadoria (20) e isto revela grau de maturidade e compromisso profissional dos membros.

Ao interpretar os dados coletados, nota-se o quão subjetiva pode ser a interpretação do material reunido. O Quadro 1 apresenta os resultados das assertivas submetidas aos respondentes, na forma de questionário, que evidenciam a existência de características de comunidade comunidades de prática na REDARTE/RJ.

Para cada uma das três características de comunidades de prática foram associados indicadores definidos por Wenger (1998). Ressalta-se que os fatores indicativos de CoP são intangíveis, podendo estar presente em mais de uma característica.

Quadro 1 - Características x Indicadores de comunidades de práticas $x$ assertivas (REDARTE/RJ) 


\begin{tabular}{|c|c|c|c|}
\hline $\begin{array}{c}\text { Características de CoP } \\
\text { (Wenger; McDermott; } \\
\text { Snyder, 2002) }\end{array}$ & Indicadores de CoP (Wenger, 1998) & Assertivas (REDARTE/RJ) & $\begin{array}{l}\text { Frequência } \\
\text { Relativa }\end{array}$ \\
\hline \multirow{6}{*}{ O Domínio } & \multirow{2}{*}{$\begin{array}{l}\text { Conhecer o que os outros sabem, o que eles podem } \\
\text { fazer, e como eles podem contribuir para uma } \\
\text { empresa; }\end{array}$} & $\begin{array}{l}\text { Acredito REDARTE/RJ criou uma imagem/marca na área de } \\
\text { Informação. }\end{array}$ & $81 \%$ \\
\hline & & $\begin{array}{l}\text { Considero o Estatuto da REDARTE/RJ é norteador dos padrões } \\
\text { da Rede. }\end{array}$ & $86 \%$ \\
\hline & \multirow{4}{*}{$\begin{array}{l}\text { Um discurso compartilhado refletindo uma certa } \\
\text { perspectiva sobre o mundo; }\end{array}$} & $\begin{array}{l}\text { A REDARTE/RJ procura parceria com outros órgãos de atuação } \\
\text { de profissionais de informação. }\end{array}$ & $81 \%$ \\
\hline & & $\begin{array}{l}\text { Possuir um planejamento estratégico para o direcionamento a } \\
\text { médio/longo prazo. }\end{array}$ & $76 \%$ \\
\hline & & $\begin{array}{l}\text { Existe reconhecimento por parte dos meus superiores da } \\
\text { importância de minha participação na REDARTE/RJ. }\end{array}$ & $85 \%$ \\
\hline & & $\begin{array}{l}\text { Participar da REDARTE/RJ dá maior visibilidade para minha vida } \\
\text { profissional. }\end{array}$ & $91 \%$ \\
\hline \multirow{6}{*}{ A Comunidade } & $\begin{array}{l}\text { A sustentabilidade das relações mútuas - } \\
\text { harmoniosas ou conflituosas; }\end{array}$ & Sinto-me comprometido com a REDARTE/RJ. & $86 \%$ \\
\hline & $\begin{array}{l}\text { Sobreposição substancial entre as descrições de } \\
\text { quem pertence dos participantes; }\end{array}$ & $\begin{array}{l}\text { Percebo que o compromisso, colaboração e o compartilhamento é } \\
\text { uma regra implícita nas ações da REDARTE/RJ. }\end{array}$ & $76 \%$ \\
\hline & Identidades mutuamente definidas; & $\begin{array}{l}\text { Sou incentivada a compartilhar com os membros da rede as falhas/ } \\
\text { as ações e atitudes incorretas. }\end{array}$ & $86 \%$ \\
\hline & $\begin{array}{l}\text { A capacidade de avaliar a adequação das ações e } \\
\text { produtos; }\end{array}$ & $\begin{array}{l}\text { Os membros da REDARTE/RJ conhecem e reconhecem o seu } \\
\text { papel. }\end{array}$ & $73 \%$ \\
\hline & A sabedoria local, histórias compartilhadas, etc. & $\begin{array}{l}\text { Existe entre os membros da REDARTE/RJ um clima de confiança } \\
\text { e credibilidade na informação gerada e compartilhada. }\end{array}$ & $81 \%$ \\
\hline & $\begin{array}{l}\text { Certos estilos reconhecidos como a indicação de } \\
\text { membros; }\end{array}$ & $\begin{array}{l}\text { As tomadas de decisões na REDARTE/RJ são pautadas em } \\
\text { definições dos membros da rede. }\end{array}$ & $85 \%$ \\
\hline \multirow{7}{*}{ A Prática } & \multirow{5}{*}{$\begin{array}{l}\text { Formas compartilhada de engajar-se e fazer as } \\
\text { coisas juntos; }\end{array}$} & Oriento meus usuários a utilizarem os recursos da REDARTE/RJ. & $72 \%$ \\
\hline & & $\begin{array}{l}\text { Posso compartilhar minhas dúvidas e incertezas que acharei } \\
\text { orientação na Rede para melhor conduzir a situação. }\end{array}$ & $86 \%$ \\
\hline & & $\begin{array}{l}\text { Sinto-me como parte responsável pelos resultados da } \\
\text { REDARTE/RJ. }\end{array}$ & $82 \%$ \\
\hline & & $\begin{array}{l}\text { A participação na REDARTE/RJ facilitar o processo de } \\
\text { empréstimo de documentos para meus usuários. }\end{array}$ & $77 \%$ \\
\hline & & $\begin{array}{l}\text { Os resultados positivos são disseminados de forma que possam } \\
\text { ser reproduzidos pelos membros em ocasião oportuna. }\end{array}$ & $81 \%$ \\
\hline & \multirow{2}{*}{$\begin{array}{l}\text { Ferramentas especificas, representações e outros } \\
\text { artefatos; }\end{array}$} & $\begin{array}{l}\text { A divulgação/disseminação da informação em Arte na } \\
\text { REDARTE/RJ possibilita manter-me atualizado. }\end{array}$ & $67 \%$ \\
\hline & & $\begin{array}{l}\text { O site da REDARTE/RJ atende as necessidades informacionais } \\
\text { dos usuários de minha instituição. }\end{array}$ & $68 \%$ \\
\hline
\end{tabular}

Fonte: Elaboração das autoras

A identificação das características de Comunidades de Prática junto à REDARTE/RJ foi um processo que resultou da mensuração das mesmas por meio das assertivas de maior ocorrência entre os respondentes. Desta forma, no Gráfico 1, apresentamos a incidência quantitativa de cada uma das características evidenciadas pela média das assertivas registradas em cada bloco. Ou seja, a frequência relativa para o Domínio foi referendada, em média, por $83,3 \%$ dos respondentes; a característica Comunidade foi referendada, em média, por $81,2 \%$ dos respondentes; e a Prática foi referendada por $76,1 \%$.

Gráfico1 - Percentual de assertivas por características de CoP existente na REDARTE/RJ 


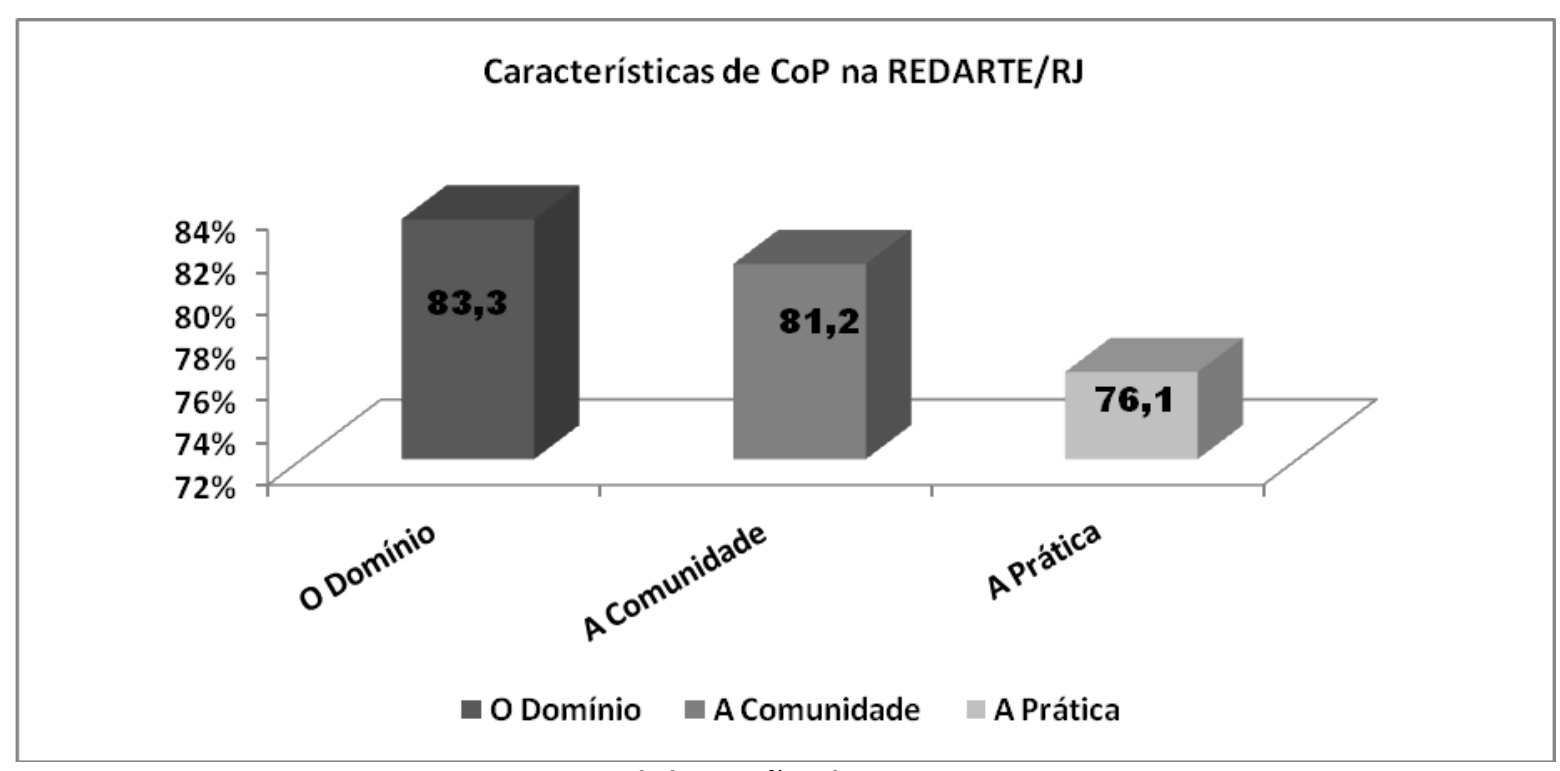

Fonte: Elaboração das autoras

\section{Considerações finais}

O presente artigo é um relato da pesquisa que buscou identificar as características qualitativas de CoP presentes nas atividades da REDARTE/RJ.

A estratégia metodológica aqui empregada nos mostrou ainda que ao estabelecer parcerias, novas oportunidades de divulgar o trabalho realizado pela rede aparecem. Legitima a comunidade por afirmar seus propósitos trazendo valor para os membros e as partes interessadas, conforme Wenger (1998, p. 125-126).

Muitas das atividades, a cumplicidade, a confiança, o comprometimento, a troca de experiências, informações e conhecimento, a colaboração e a interação dos membros da rede são características encontradas em comunidades de prática, ao mesmo tempo são aspectos que contribuíram para o sucesso da rede.

Estudar as características de comunidades de prática em relação às atividades da REDARTE/RJ certifica que é possível o aprendizado coletivo e que a troca de experiência acontece mesmo em se tratando de instituições integrantes de área de conhecimento tão distintas.

Verificou-se que as características de comunidades de prática existentes na REDARTE/RJ são indícios de que a rede promove com suas atividades a gestão do conhecimento organizacional entre os profissionais da informação em Arte.

\section{Referências}

AMARAL, Sueli Angélica. Web sites: uso de tecnologias no cumprimento das funções da biblioteca. Informação \& Sociedade, João Pessoa, v. 15, n. 2 , p. 15-40, jul./dez. $2005 . \quad$ Disponível em: http://www.ies.ufpb.br/ojs/index.php/ies/article/view/30/1511. Acesso em: 5 dez. 2014. 
ARAGON, Claudia. A REDARTE/RJ: entrevista. Rio de Janeiro, 9 abril 2014. Entrevista concedida a Elisete Melo.

FERREZ, Helena Dodd. Uma experiência brasileira em rede de bibliotecas de arte: a REDARTE. In: CONFERENCE OF THE ART LIBRARIES SOCIETY OF NORTH AMERICA. ARLIS/NA, 28, 2000. Pittsburg: ARLIS/NA, 2000. Disponível em: http://redarterj.com/wp-content/uploads/2011/12/Umexperiencia-em-Rede-de-Bibliotecas.-Helena-Ferrez.pdf. Acesso em: 15 fev. 2014.

INTERNATIONAL FEDERATION OF LIBRARY ASSOCIATIONS AND INSTITUTIONS. Knowledge Management Section. What is Knowledge Management? Disponível em: http://iflakm.wikispaces.com/What+is+Knowledge+Management\%3F.

Acesso em: 28 Jul. 2014.

MELO, Elisete de S. Comunidades de prática: um estudo de caso na Rede de Bibliotecas e Centros de Informação em Arte no Estrado do Rio de Janeiro - REDARTE/RJ. 2015. 128 f. Dissertação (Mestrado Profissional em Biblioteconomia) - Universidade Federal do Estado do Rio de Janeiro. 2015.

MINTZBERG, Henry; AHLSTRAND, Bruce; LAMPEL, Joseph. Safari de estratégia: um roteiro pela selva do planejamento estratégico. 2. ed. Porto Alegre: Bookman, 2010.

NONAKA, Ikujiro; TAKEUCHI, Hirotaka. Criação do conhecimento na empresa: como as empresas japonesas geram a dinâmica da inovação. 2 . ed. Rio de Janeiro: Campus, 1997.

REDES DE BIBLIOTECAS E CENTROS DE INFORMAÇÃO EM ARTE ARTE NO ESTADO DO RIO DE JANEIRO - REDARTE/RJ. Atas. Rio de Janeiro, 19951997, 2005-2007, 2010-2014. Mimeo.

REDES DE BIBLIOTECAS E CENTROS DE INFORMAÇÃO EM ARTE NO ESTADO DO RIO DE JANEIRO - REDARTE/RJ. Estatuto. Rio de Janeiro, 2011. Disponível em: http://redarterj.com/estatuto/. Acesso em: 26 jan. 2015.

TERRA, J. Claudio C. Gestão do Conhecimento: 7 dimensões e 100 práticas gerenciais. $2007 . \quad$ Disponível em: http://biblioteca.terraforum.com.br/BibliotecaArtigo/GC 7 Dimens\%C3 \%B5es e 100 pr\%C3\%A1ticas gerenciais.pdf . Acesso em: 06 jun. 2014.

WENGER, Etienne. Communities of practice: learning, meaning, and identity. Cambridge, UK: University of Cambridge Press, 1998.

WENGER, Etienne. Communities of Practice and Social Learning Systems. Organization, v. 7, p. 225-246, May, 2000. Disponível em: TTP://org.sagepub.com/content/7/2/225. abstract. Acesso em: 10 dez. 2013. 
WENGER, Etienne; MCDEMORTT, Richard; SNYDER, William. Cultivating communities of practice: a guide to managing knowledge. Boston: Harvard Business Press, 2002. 284 p.

WENGER, Etienne. Communities of practice: the art of learning together. Apresentação. USA: Etienne Wenger: Learning for small planet, 2006.

WENGER, Etienne. Communities of practice and social learning systems: the career of a concecpt. 2010. Disponível em: http://wengertrayner.com/wp-content/uploads/2012/01/09-10-27-CoPs-and-systemsv2.0.pdf. Acesso em: $21 \mathrm{dez} .2013$. 